\title{
Hypofractionated Radiotherapy for Post-Operative Breast Cancer Patients at Delta Hospital - an Evaluation of Clinical Experience
}

\author{
Parvin Akhter Banu${ }^{1}$, Naheed Rukhsana ${ }^{2}$, MA Jabber ${ }^{3}$, Motiur Rahman ${ }^{4}$, Sadiq R Malik ${ }^{5}$
}

\begin{abstract}
Background: As hypofractionated radiotherapy for post-operative breast cancer patients safe, effective and more convenient, it might be beneficial for patients of developing countries like ours. Objective: This study was done to evaluate the safety and efficacy of hypofractionated whole breast radiation therapy in patients who underwent breast conserving surgery and hypofractionated radiation therapy in patients who underwent mastectomy and axillary clearance. Materials and method: This cross sectional study was conducted in Delta Hospital Ltd, Dhaka, Bangladesh, including 50 postoperative patients, (12 patients in Breast Conservation Therapy group and 38 in Post Mastectomy Radiation Therapy group), with invasive ductal carcinoma of breast treated with this hypofractionated radiotherapy protocol during the last 1.5 year. The patients were treated with 3DCRT, LINAC, $6 \mathrm{MV}$ photon and appropriate electron energy. Results: Minimal post treatment acute morbidity was observed. Forty seven patients (94\%) had grade-I acute skin toxicity and only 3 patients (6\%) developed grade-II acute skin toxicity. Conclusion: Hypofractionated radiotherapy is as safe and effective as conventional fractionated radiotherapy and superior in terms of convenience.
\end{abstract}

Keywords: Radiotherapy; hypofractionated radiotherapy; breast cancer.

$$
\text { Delta Med Col J. Jan 2015;3(1):4-8 }
$$

\section{Introduction}

Lumpectomy or breast conserving surgery and post-operative breast irradiation has been established as a standard care for early breast cancer. According to the National Comprehensive Cancer Network (NCCN) Guidelines 2.2013 for invasive breast cancer, the recommended dose and number of fractions are conventional fraction whole breast irradiation (CF-WBI) - 45-50 Gy in 1.8-2 Gy per fraction, or hypofraction whole breast irradiation (HF-WBI) - 42.5 Gy at 2.66 Gy per fraction. A boost to the tumor bed is recommended in patients at higher risk for local failure, (age $<50$ years, positive axillary nodes, lymphovascular invasion, or close margins). Typical doses are 10-16 Gy at 2 Gy per fraction. All dose schedules are given 5 days per week. However, the recommendation of 1.8-2 Gy per fraction is simply based on the history of radiation therapy instead of results of clinical trials that researched the optimal single dose. ${ }^{1}$

Post mastectomy regional radiotherapy (PMRT) is effective at preventing locoregional failure (LRF) and thereby increasing relapse-free and overall survival rates, particularly in patients with more than three involved axillary lymph nodes. ${ }^{2}$

1. Chief Consultant, Oncology, Delta Hospital Ltd., Dhaka, Bangladesh.

2. Consultant, Oncology, Delta Hospital Ltd., Dhaka, Bangladesh.

3. Principal Physicist, Delta Hospital Ltd., Dhaka, Bangladesh.

4. Senior Radiation Oncology Physicist, Delta Hospital Ltd., Dhaka, Bangladesh.

5. Chief Radiation Oncology Physicist, Delta Hospital Ltd., Dhaka, Bangladesh.

Correspondence: Dr. Parvin Akhter Banu. e-mail: pabanu@yahoo.com 
A number of large randomized trials have been conducted in different parts of the world by different study groups. ${ }^{3-11}$ Some of them are given below in tabulated form.

\begin{tabular}{lcccc}
\hline Study group & Number of cases & $\begin{array}{c}\text { Fractionated } \\
\text { dose (Gy/Fr/Days) }\end{array}$ & $\begin{array}{c}\text { Median follow-up } \\
\text { (years) }\end{array}$ & IBTR rate \\
\hline Canada $^{3}$ & \multirow{2}{*}{$612 / 622$} & $42.5 / 16 / 22$ & 12 & $7.4 \%$ \\
& & $50 / 25 / 35$ & & $7.5 \%$ \\
RMH/GOC $^{4}$ & $474 / 622 / 470$ & $39 / 13 / 35$ & 9.7 & $15 \%$ \\
& & $42.9 / 13 / 35$ & & $9.6 \%$ \\
START A $^{5}$ & \multirow{2}{*}{$737 / 750 / 749$} & $50 / 25 / 35$ & 5.1 & $12 \%$ \\
& & $39 / 13 / 35$ & & $4.6 \%$ \\
& & $41.6 / 13 / 35$ & & $3.2 \%$ \\
START B $^{6}$ & \multirow{2}{*}{$1110 / 1105$} & $50 / 25 / 35$ & 6.0 & $3.2 \%$ \\
& & $40 / 15 / 21$ & & $2.0 \%$ \\
& & $50 / 25 / 35$ & & $3.3 \%$ \\
\hline
\end{tabular}

Hypofractionation post-mastectomy radiation therapy (HF-PMRT) studies have shown it is as safe and as effective as conventional PMRT. HF-WBI is a preferred choice in Western Countries like USA, Canada and UK because of its economic and geographical convenience. In USA, breast conserving therapy (BCT) that consists of $50 \mathrm{~Gy}$ in 25 fractions over 5 weeks is commonly applied for breast conservation after lumpectomy. No significant statistical differences were observed in recurrence-free rates, cosmetic outcomes and late adverse event between the HF-WBI group and the CF-WBI group in the randomized trial. A task force authorized by the American Society for Radiation Oncology (ASTRO) developed an evidence based guideline in 2010 and recommended the use of HF-PMRT in early stage breast cancer. ${ }^{3-11}$

\section{Social Background}

Most breast cancer patients who receive post-operative irradiation are outpatients and, therefore, treatment that requires 5 days a week over 5 weeks may cause considerable economic/time burden on patients. In addition, despite the rapid increase of overall number of patients subjected to radiation therapy, department of radiation oncology are only able to provide treatment to a limited number of patients in the current situation, due to manpower/ time/equipment constraints. Meanwhile, if the
HF-WBI and HF-PMRT are adopted as one of the standard radiation therapies in Asian countries, the following benefits will be gained:

- Less number of fractions can reduce the time and economic burden on patients.

- Medical institutions can provide radiation therapy to more patients and, consequently, they can increase their income from radiation treatment.

\section{Materials and method \\ HF-WBI}

For cases without high grade factors 43.2 Gy to the whole breast was given in 16 fractions over 22 days. For patients with high grade factors (age < 50 years, positive axillary nodes, lymphovascular invasion, or close margins) - 43.2 Gy to the whole breast in 16 fractions over 22 days with an additional tumour bed boost of 8.1 Gy in 3 fractions were given.

Irradiation position: Patients were immobilized with devices in the supine position with the upper limb of affected side (both sides of upper limbs) raising.

Radiation sources: $6 \mathrm{MV}$ x-ray to the whole breast, and electron to the single-field tumour bed boosts.

Irradiation methods: Tangential irradiation method that aligns posterior margins (a field in field method, wedge filter) for WBI and single-field irradiation for boosts. Planning to achieve target dose homogeneity within $\pm 7 \%$ of planned treatment volume (PTV).

\section{Radiation field for WBI}

Inner margin - midline of the sternum. Outer margin - middle axillary line or 1.5-2 cm outside the palpable mammary glands. Upper margin between the upper edge of the acromial extremity of clavicle and the lower edge of the extremitas sternalis claviculae. Lower margin - 1 to $2 \mathrm{~cm}$ from the lower edge of the breast. The supraclavicular fossa and internal mammary lymph nodes were not included. The thickness of the lung field within the radiation field should not exceed $3 \mathrm{~cm}$. 


\section{HF-PMRT}

For cases with negative surgical margins - 43.2 Gy to the chest wall and supraclavicular region was given in 16 fractions over 22 days.

Radiation sources: LINAC, $6 \mathrm{MV}$-ray to the chest wall and supraclavicular region.

Irradiation methods: Tangential irradiation method that aligns posterior margins (a field in field method, wedge filter) for chest wall and single or opposed fields irradiation for supraclavicular fossa. Planning to achieve target dose homogeneity within $\pm 7 \%$ of PTV.

\section{Radiation field for chest wall}

Inner margin - midline of the sternum. Outer margin - middle axillary line. Upper margin between the upper edge of the acromial extremity of clavicle and the lower edge of the extremitus sternalis claviculae. Lower margin - 1 to $2 \mathrm{~cm}$ from the lower edge of the contralateral breast. The internal mammary lymph nodes were not included. The thickness of the lung field within the radiation field should not exceed $3 \mathrm{~cm}$.

This cross sectional study was carried out in Delta Hospital Limited. A total of 50 postoperative patients, (12 patients in BCT group and 38 in PMRT), with invasive ductal carcinoma of breast have been treated with this hypofractionated radiotherapy protocol during the last 1.5 year. All the patients were treated with 3DCRT, LINAC, 6 MV photon and appropriate electron energy. Age range of the patients was 29-65 years. Written consents were taken from all patients.

Figures showing planning of radiotherapy with dosimetry (Fig. 1a, b, c \& d).

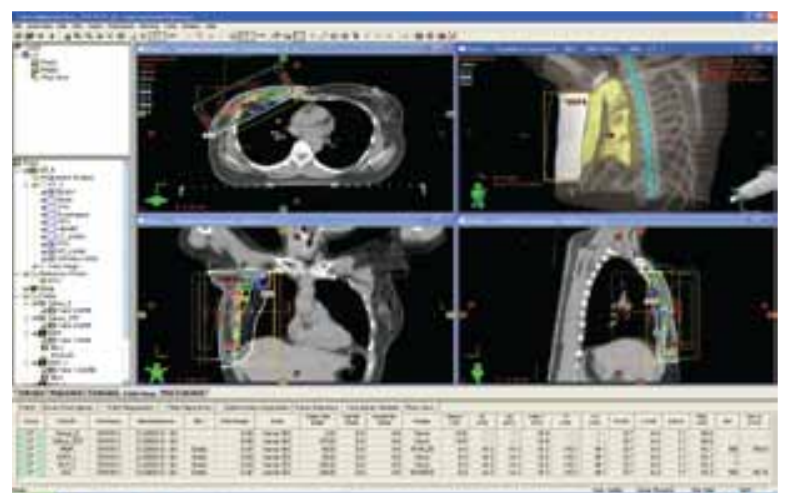

Fig. 1(a): Radiotherapy planning for post-mastectomy cases

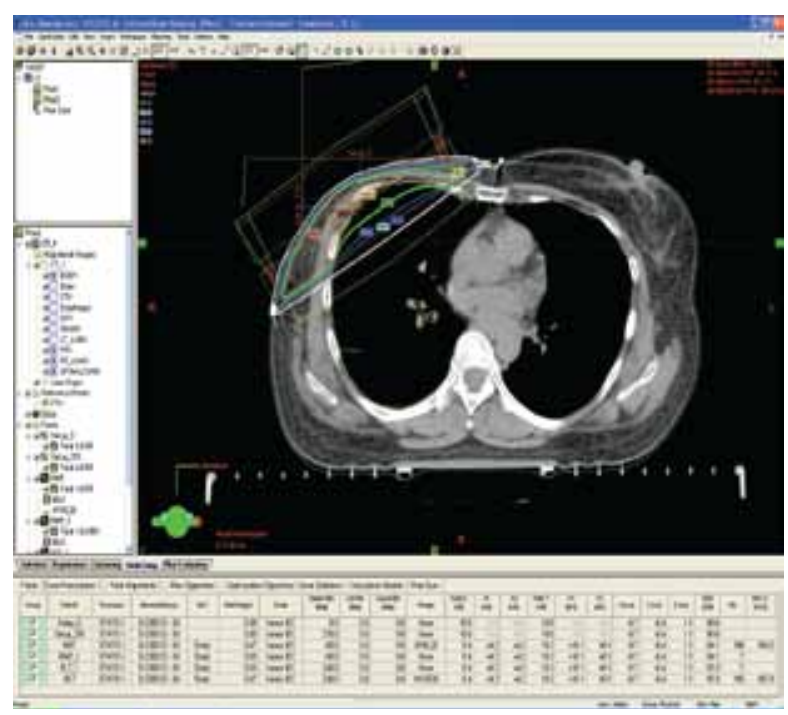

Fig. 1(b): Radiotherapy planning for post-mastectomy cases

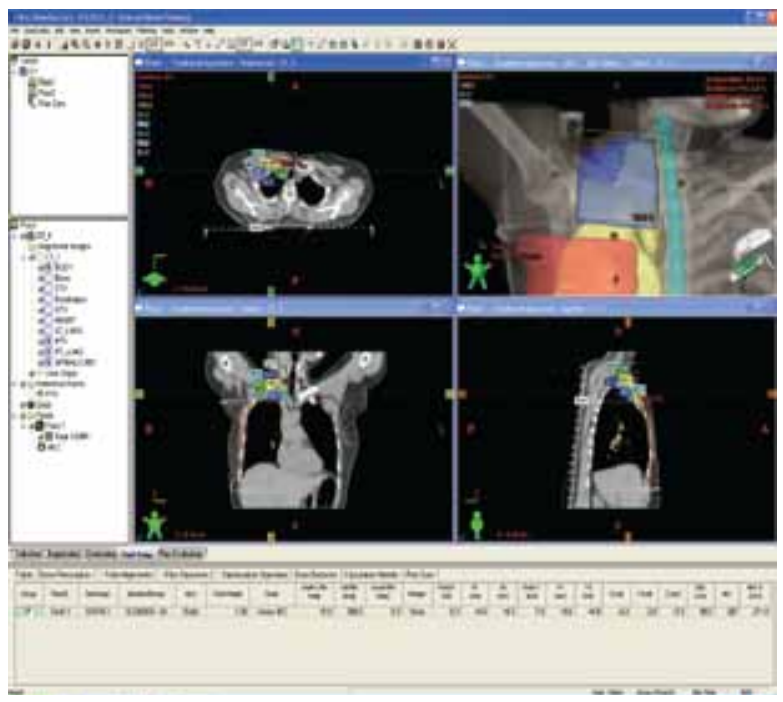

Fig. 1(c): Radiotherapy planning for supraclavicular fossa

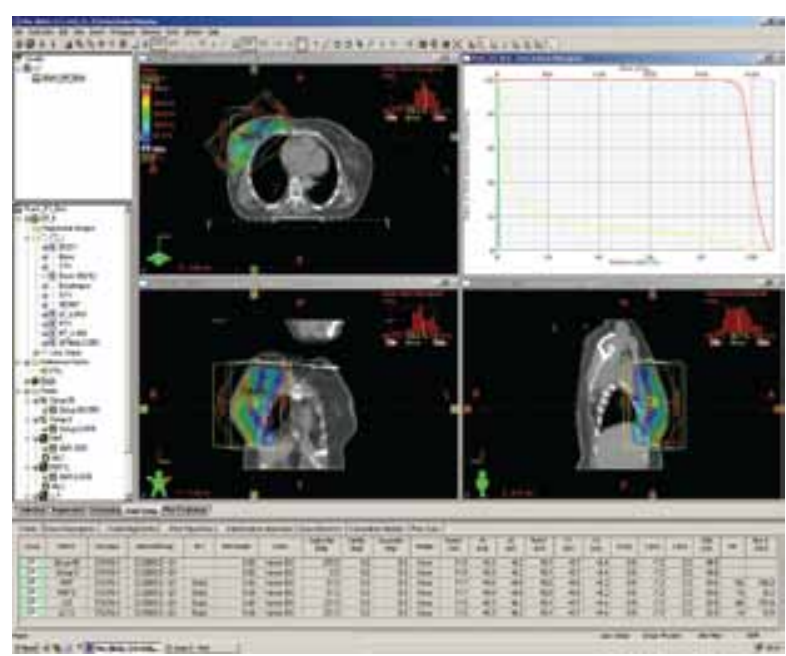

Fig. 1(d): Radiotherapy planning for Breast Conservation Surgery (BCS) 


\section{Results}

Characteristic features of patients are given below (Table I).

Table I: Patient characteristics $(\mathbf{N}=\mathbf{5 0})$

\begin{tabular}{|c|c|c|}
\hline Variables & Frequency & Percentage \\
\hline \multicolumn{3}{|l|}{ Age group in years } \\
\hline $29-40$ & 12 & 24 \\
\hline $41-50$ & 24 & 48 \\
\hline $51-65$ & 14 & 28 \\
\hline \multicolumn{3}{|l|}{ Stage of breast carcinoma } \\
\hline Stage IA & 5 & 10 \\
\hline Stage IB & 2 & 4 \\
\hline Stage IIA & 26 & 52 \\
\hline Stage IIB & 17 & 34 \\
\hline \multicolumn{3}{|l|}{ Treatment pattern } \\
\hline PMRT & 38 & 76 \\
\hline $\mathrm{BCT}$ & 12 & 24 \\
\hline \multicolumn{3}{|l|}{ Histology* and Grading } \\
\hline Grade 1 & 5 & 10 \\
\hline Grade II & 30 & 60 \\
\hline Grade III & 15 & 30 \\
\hline \multicolumn{3}{|l|}{ Receptor status } \\
\hline $\mathrm{ER}+\mathrm{ve}$ & 25 & 50 \\
\hline $\mathrm{PR}+\mathrm{ve}$ & 22 & 44 \\
\hline Her-2 (3+) & 12 & 24 \\
\hline Unknown & 1 & 2 \\
\hline Completed Chemotherapy & $49 * *$ & 98 \\
\hline Completed EBRT & 50 & 100 \\
\hline
\end{tabular}

* Histologically all the subjects had invasive ductal carcinoma

** One patient did not complete scheduled chemotherapy

Acute and late radiation toxicity and morbidity were assessed and graded according to toxicity criteria of the Radiation Therapy Oncology Group (RTOG) and the European Organization for Research and Treatment of Cancer (EORTC). ${ }^{12,13}$ Patients developed minimal post treatment acute morbidity. All of them developed grade I fatigue. Forty seven patients (94\%) had grade-I acute skin toxicity and only 3 patients $(6 \%)$ developed grade-II acute skin toxicity. All the patients are in regular follow-up and till now no grade II-III late morbidity seen in any of the patients.

\section{Discussion}

The concept of Hypofractionation (HF), originally conceived in the 1960s, was initially associated with a high rate of late complications, therefore resulting in abandonment of its use. As the understanding of the radiobiologic principles that govern normal tissue responses improved, HF regimens regained popularity in the United States. To date, there have been several randomized trials that have established equivalent local control and cosmetic outcomes between HF and conventional fractionation (CF) regimens. ${ }^{2}$ Evidence based guideline by ASTRO also recommended its use in early stage breast cancer however they could not agree on the appropriateness of a tumour bed boost in patients treated with HF-WBI. ${ }^{8}$

In this study there was minimal radiation related complication in terms of fatigue and acute skin toxicity. Our short term outcomes are in consistence with several other studies. A study done by Ciammella et al. ${ }^{14}$ revealed comparable results - grade I skin toxicity was observed in 79\% patients and grade II skin toxicity in $12 \%$ of patients. These proportions were $80 \%$ and $5 \%$ in the clinical trial carried out by Janssen et al. ${ }^{15}$ which are also almost similar to that of ours. All the patients are under regular follow up and none of them has developed any of the radiation related late morbidities yet. Though till date no other complications documented, still it is too early to evaluate for efficacy in our patients.

Hypofractionation in breast cancer is an issue that can have widespread implications in early stage breast cancer treatment throughout the world. If found to have equivalent cosmesis, locoregional control, and survival to standard doses and schedules, it might be a remarkable achievement in perspective of our country, in treating the larger number of patients overcoming socio economic restrains, shortage of trained and expert manpower and also scarcity of specialized oncology centres. Unfortunately, the demonstration of all of these would need follow-up data nearing 15 years. For now, the general acceptance of hypofractionation in breast cancer hangs in the balance. 


\section{References}

1. NCCN [Internet]. NCCN Clinical Practice Guidelines in Oncology. 2013 [cited 2014 Aug 12]. Available from: http://www.nccn.org/professionals/ physician_gls/f_guidelines.asp.

2. Yang TJ, Ho AY. Radiation Therapy in the Management of Breast Cancer. Surg Clin N Am. 2013;93:455-71.

3. Whelan TJ, Pignol JP, Levine MN, Julian JA, MacKenzie R, Parpia S, et al. Long-Term Results of Hypofractionated Radiation Therapy for Breast Cancer. N Engl J Med. 2010;362:513-20.

4. Owen JR, Ashton A, Bliss JM, Homewood J, Harper C, Hanson J, et al. Effect of Radiotherapy Fraction Size on Tumor Control in Patients with Early Stage Breast Cancer after Local Tumor Excision: Long-Term Results of a Randomized Trial. Lancet Oncol. 2006;7:467-71.

5. Bentzen SM, Agrawal RK, Aird EG, Barrett JM, Barrett-Lee PJ, Bliss JM, et al. The UK Standardization of Breast Radiotherapy (START) Trial A of Radiotherapy Hypofractionation for Treatment of Early Breast Cancer: A Randomized Trial. Lancet Oncol. 2008;9(4):331-41.

6. Bentzen SM, Agrawal RK, Aird EG, Barrett JM, Barrett-Lee PJ, Bliss JM, et al. The UK Standardization of Breast Radiotherapy (START) Trial B of Radiotherapy Hypofractionation for Treatment of Early Breast Cancer: A Randomized Trial. Lancet. 2008;371:1098-107.

7. Karasawa K, Kunogi H, Hirai T, Hojo H, Hirowatari $\mathrm{H}$, Izawa $\mathrm{H}$, et al. Comparison of Hypofractionated and Conventionally Fractionated Whole-Breast Irradiation for Early Breast Cancer Patients: A Single-Institute Study of 1,098 Patients. Breast Cancer. 2014;21(4):402-408.

8. Smith BD, Bentzen SM, Correa CR, Hahn CA, Hardenbergh PH, Ibbott GS, et al. Fractionation for Whole Breast Irradiation: An American Society for
9. Radiation Oncology (ASTRO) Evidence-Based Guideline. Int $\mathrm{J}$ Radiat Oncol Biol Phys. 2011;81:59-68.

10. Eldeeb H, Awad I, Elhanafy O. Hypofractionation in Post-Mastectomy Breast Cancer Patients: Seven-Year Follow-Up. Med Oncol. 2012;29(4):2570-76.

11. Pinitpatcharalert A, Chitapanarux I, Euathrongchit J, Tharavichitkul E, Sukthomya V, Lorvidhaya V. A Retrospective Study Comparing Hypofractionated Radiotherapy and Conventional Radiotherapy in Post Mastectomy Breast Cancer. J Med Assoc Thai. 2011;94 Suppl 2:S94-102.

12. Deantonio L, Gambaro G, Beldì D, Masini L, Tunesi S, Magnani C, et al. Hypofractionated Radiotherapy after Conservative Surgery for Breast Cancer: Analysis of Acute and Late Toxicity. Radiation Oncology. 2010;5:112 .

13. Wikibooks [Internet]. RTOG/EORTC Radiation Toxicity Grading. 2014 Jan 21 [cited 2014 Aug 12]. Available from: http://en.wikibooks.org/wiki/ Radiation_Oncology/Toxicity_grading/RTOG.

14. Rubin P, Constine LS, Fajardo LF, Phillips TL, Wasserman TH. RTOG Late Effects Working Group. Overview of Late Effects Normal Tissues (LENT) Scoring system. Radiotherapy Oncology. 1995;35: 9-10.

15. Ciammella P, Podgornii A, Galeandro M, Micera R, Ramundo D, Palmieri T, et al. Toxicity and Cosmetic Outcome of Hypofractionated Whole-Breast Radiotherapy: Predictive Clinical and Dosimetric Factors. Radiation Oncology. 2014;9:97.

16. Janssen S, Glanzmann C, Lang S, Verlaan S, Streller T, Wisler D, et al. Hypofractionated Radiotherapy for Breast Cancer Acceleration of the START A Treatment Regime: Intermediate Tolerance and Efficacy. Radiation Oncology. 2014;9:165. 